\title{
Active vibration control of composite shallow shells: An integrated approach
}

\author{
N. Rahman ${ }^{1}$, M. N. Alam ${ }^{1}$ and M. Junaid ${ }^{1}$ \\ ${ }^{1}$ Department of Mechanical Engineering, \\ Zakir Husain College of Engineering and Technology, \\ Aligarh Muslim University, Aligarh, U.P-202002, India. \\ *Email: najeebalig@ rediffmail.com
}

\begin{abstract}
Active vibration control of smart composite shallow shells with distributed piezoelectric sensors and actuators is presented in this work. An integrated approach is used for recording the uncontrolled and controlled vibration response under pressure impulse load. The FE model developed in ABAQUS is utilized to generate the global mass, stiffness and load matrices of the system. The system matrices are arranged in statespace format and the dynamic equations of the system are obtained. The controlled responses are achieved using the inputs from FE model in ABAQUS in conjunction with the developed MATLAB codes for Constant Gain Velocity Feedback (CGVF) and Linear Quadratic Regulator (LQR) control strategies. The method is first validated by comparing the natural frequencies obtained using the ABAQUS generated matrices with that obtained using an FE model with four noded quadrilateral shallow shell element based on efficient zigzag theory. The shell element uses the concept of electric nodes to satisfy the equipotential condition of electrode surface. An 8-noded linear piezoelectric brick element is used for piezoelectric layers and an 8-noded quadrilateral continuum shell element is used for the elastic layers of hybrid shells for making the finite element mesh in ABAQUS. The non-dimensional natural frequencies and active vibration control responses for hybrid composite cylindrical and spherical shells are presented for clamped-clamped and cantilever boundary conditions. Boundary conditions have significant effect on vibration amplitude, control voltage and settling time. In comparison to CGVF controller, a better control in lesser time is achieved with LQR controller for a shell with similar boundary conditions. Larger gain values $(G)$ are required for vibration control of thick shells.
\end{abstract}

Keywords: Shell; composite; finite element modeling; ABAQUS; constant gain velocity feedback; linear quadratic regulator.

\section{INTRODUCTION}

Laminated composite structures have been widely used for satellites, aerospace, robotics, and similar other applications. Vibration suppression in these structures has received enormous research attention in recent years. Active damping control has the ability to provide adjustable and significant damping that traditional passive damping treatments cannot. Qian et al. [1] studied the active vibration control of composite laminated shells via embedded magnetostrictive layers. They used a higher order layerwise theory to estimate the coupled strain and stresses accurately. A shear deformable shell element based on Reissner's hypothesis for the analysis of smart 
laminated composite shells has been developed by Bhattachariya et al. [2]. They adopted an IMSC based LQR control methodology for the active vibration control of laminated spherical shell. Balamurugan and Narayanan [3] formulated a nine-noded piezolaminated shell finite element for modeling and analysis of composite shell structures with distributed piezoelectric sensors and actuators. This element is developed by using the degenerate solid approach. Ray [4] presented an analytical solution based on the coupled CLT for active vibration control of simply supported cylindrical shells. Kapuria and Yasin [5] studied the active vibration suppression of smart shallow shells using a four-node quadrilateral shell element based on a fully coupled efficient layerwise (zigzag) theory. Ray and Reddy [6] demonstrated the active vibration control of laminated cylindrical shells using optimally placed patches of active constrained layer damping treatment. Kumar et al. [7] numerically evaluated the influence of actuator damage on the performance of closed loop vibration control. They considered debonding a damage mode. Zhang et al. [8] developed a novel laminated piezoelectric actuator to control the vibration of a cylindrical shell structure. Rahman and Alam [9] presented vibration suppression of smart beams using the piezoelectric patch structure. They developed an experimental set-up to obtain the active vibration suppression of smart beam. Li et al. [10] discussed the control of the piezoelastic laminated cylindrical shell's vibration under hydrostatic pressure. Kapuria and Yasin [11] presented an efficient finite element (FE) model for the active vibration control response of smart laminated beams integrated with electroded piezoelectric sensors and actuators. Lee and Reddy [12] developed the third-order shear deformation theories of laminated composite shells using the strain-displacement relations of Donnell and Sanders theories.

Rahman and Alam [13, 14] studied the active vibration suppression of smart hybrid beam using a two-noded finite element based on zigzag theory. Moita et al. [15] presented a finite element formulation for active vibration control of thin plate laminated structures with piezoelectric sensors and actuators. Ray and Mallik [16] theoretically investigated the effectiveness of piezoelectric fiber reinforced composite material in the development of new actuators as element of smart structures. Pradhan and Reddy [17] presented the analytical solutions of laminated composite shells with embedded actuating layers. Ray and Batra [18] analysed active constrained layer damping of functionally graded shells under a thermal environment and investigated the performance of PZCs as materials for the constraining layer of the ACLID treatment. Zhang et al. [19] investigated adaptive vibration control of a cylindrical shell partially covered by laminated PVDF actuator (LPA). Sarangi and Ray [20] addressed the active control of geometrically nonlinear vibrations of doubly curved functionally graded (FG) laminated composite shells integrated with a patch of active constrained layer damping (ACLD) treatment under the thermal environment. Neto et al. [21] extended a flexible multibody dynamics formulation of complex models including elastic components made of composite materials. Hua-ping et al. [22] presented a kind of active vibration control method based on active damping and optimization design for driving load of multibody system. Song and Li [23] studied the active aeroelastic flutter analysis and vibration control with the piezoelectric patches. Shah and Ray [24] performed analysis of active control of vibration of thin laminated composite truncated circular conical shells. Sarangi and Ray [25] performed the analysis of active constrained layer damping (ACLD) of geometrically nonlinear transient vibrations of doubly curved laminated composite shells. Sun and Huang [26] derived an analytical formulation for modelling the behaviour of laminated composite beams with integrated piezoelectric sensor and 
actuator. Zhang and Shen [27] presented an analytical formulation for structural vibration control of laminated plates. Baillargeon et al. [28] utilized ABAQUS in the numerical simulation of active feedback control for the purpose of vibration suppression of structural systems. Vasques and Rodrigues [29] produced a numerical active vibration control model to reduce the mechanical vibrations of the system by modification of the systems structural response. Balamurugan and Narayanan [30] dealt with the active vibration control of smart/intelligent shells with a distributed piezoelectric sensor and actuator layer bonded onto the top and bottom surfaces of the structures. Roy and Chakraborty [31] presented an improved genetic algorithm (GA) based optimal vibration control of smart fiber reinforced polymer (FRP) composite shell structures. Bailey and Hubbard [32] conducted the active vibration control on a thin cantilever beam through the distributed piezoelectric-polymer and designed the controller using Lyapunov's second and direct method. Kim et al. [33] performed the active vibration control of composite shell structure with the optimized sensor/actuator system. Sun et al. [34] designed a multimodal fuzzy sliding mode controller to provide active vibration control of conical shell structure. Loghmani et al. [35] theoretically and experimentally studied the active vibration control of a cylindrical shell using piezoelectric disks. He and Chen [36] proposed a hybrid optimization strategy for design of piezoelectric cylindrical flat shell structure.

After exhaustive literature review, it has been observed that researchers have presented different models for vibration control based on higher order theories. Those models are quite involved and requires lots of computational efforts. In this work, a simpler integrated approach is used to obtain active vibration control response of smart composite shallow shells under pressure impulse loading. The integrated approach refers to the methodology which uses the FE model in ABAQUS to generate inputs to be used in the MATLAB environment for controlling the responses. The FE model is developed in ABAQUS to generate the global mass, stiffness and load matrices of the system and the controlled responses are obtained using these inputs in conjunction with the developed MATLAB codes for constant gain velocity feedback (CGVF) and Linear Quadratic Regulator (LQR) control strategies. Piezoelectric brick elements for piezo layers and continuum shell elements for the elastic layers are utilised for modelling in ABAQUS.The accuracy of the used elements is assessed for free vibration responses by comparing the natural frequencies obtained using the integrated approach with that obtained using a four noded quadrilateral shallow shell element based on efficient zigzag theory. The controlled responses are presented for cantilever and clampedclamped boundary conditions.

\section{FORMULATION AND METHODOLOGY}

\section{Efficient zigzag theory for hybrid piezoelectric shell}

A smart multi-layered curved shallow shell [5] is considered. The shell is shallow and its middle surface can be represented by the planform coordinates ( $x, y)$ for the rectangular orthotropy. The transverse normal stress $\sigma_{z}$ is neglected $\left(\sigma_{z} \square 0\right)$ in comparison with other stress components. Using this assumption, the linear constitutive equations relating the in-plane stresses $\sigma$ and transverse shear stresses $\tau$ to the inplane strains $\varepsilon$ and transverse shear strains $\gamma$ of a generally orthotropic piezoelectric lamina are expressed as

$$
\sigma=\bar{Q} \varepsilon-\bar{e}_{3}^{T} E_{z}, \tau=\hat{Q} \gamma-\hat{e} E, D=\hat{e}^{T} \gamma+\hat{\eta} E, D_{z}=\bar{e}_{3} \varepsilon+\bar{\eta}_{33} E_{z}
$$


For the shallow shell theory assumptions [Qatu, 2004] the strain-displacement relations for the shell reduces to

$$
\begin{gathered}
\varepsilon_{x}=u_{x, x}+w / R_{x} \quad \varepsilon_{y}=u_{y, y}+w / R_{y} \quad, \gamma_{x y}=u_{y, x}+u_{x, y}+2 w / R_{x y}, \\
\varepsilon_{z}=w, \quad \gamma_{x z}=w_{, x}+u_{x, z}, \quad \gamma_{y z}=w_{, y}+u_{y, z}, \\
E_{x}=-\phi_{, x}, \quad E_{y}=-\phi_{, y}, E_{z}=-\phi_{, z}
\end{gathered}
$$

where a subscript comma denotes partial differentiation. $u_{x}, u_{y}$ and $w$ denote the inplane and transverse displacements, and $\phi$ is the electric potential. $R_{x}$ and $R_{y}$ are radii of curvature along $x$ and $y$ directions, and $R_{x y}$ is the radius of twist.

The electric potential $\phi$ is assumed to be piecewise quadratic [5] between $n_{\phi}$ interpolation points along the thickness.

$$
\phi(x, y, z, t)=\psi_{\phi}^{j}(z) \phi^{j}(x, y, t)+\psi_{c}^{l}(z) \phi_{c}^{l}(x, y, t)
$$

The deflection $w$ is approximated by adding the explicit contribution of the electric filed due to the $d_{33}$ effect to the constant components $w_{0}$, obtained by integrating the constitutive equation $\varepsilon_{z}=w_{, z} \square-\bar{d}_{33}^{(k)} \phi_{, z}$ :

$$
w(x, y, z, t)=w_{0}(x, y, t)-\left\{\bar{\psi}_{\phi}^{j}(z) \phi^{j}(t)+\bar{\psi}_{c}^{l}(z) \phi_{c}^{l}(x, y, t)\right\}
$$

The inplane displacements are approximated by superimposing a global third order variation in $\mathrm{z}$ with a linear layerwise variation.

$$
u(x, y, z, t)=u_{0}(x, y, t)-z w_{0 d}(x, y, t)+R^{k}(z) \psi_{0}(x, y, t)
$$

where $u_{0}$ and $\psi_{0}$ denotes the translations and shear rotations of the reference surface. Eq. (5) can be rewritten in matrix form as

$$
u=f_{0}(z) \bar{u}_{1}
$$

where

$$
\bar{u}_{1}=\left[\begin{array}{llllll}
u_{0_{x}} & u_{0_{y}} & -w_{0, x} & -w_{0, y} & \psi_{0 x} & \psi_{0 y}
\end{array}\right], \quad f_{0}(z)=\left[\begin{array}{lll}
I_{2} & z I_{2} & R^{k}(z)
\end{array}\right]
$$

Substituting the displacement field expressions into the strain-displacement and electric field relations yields

$$
\varepsilon=f_{1}(z) \bar{\varepsilon}_{1}, \gamma=f_{2}(z) \bar{\varepsilon}_{2}, E=-f_{3}(z) \bar{\varepsilon}_{3}, E_{z}=-f_{4}(z) \bar{\varepsilon}_{4}
$$

Let $p_{z}^{1}, p_{z}^{2}$ be the normal forces per unit area applied on the bottom and top surfaces of the shell and $\rho^{k}$ be the material mass density of kth layer. The extended Hamilton's principle for the shallow shell can be expressed as, using notation

$$
<\ldots>=\sum_{k=1}^{L} \int_{z_{k-1}^{+}}^{\bar{z}_{k-1}^{k}}(\ldots) d z
$$

for integration along the thickness, as

$$
\begin{gathered}
\int\left[<\rho^{k}\left(\delta u^{T} \ddot{u}+\ddot{w} \delta w\right)+\delta \varepsilon^{T} \sigma+\delta \gamma^{T} \tau-\delta E^{T} D-D_{z} E_{z}>\right. \\
+\left(1+\frac{z_{0}}{R_{x}}\right)\left(1+\frac{z_{0}}{R_{y}}\right) p_{z}{ }^{1} \delta w\left(x, y, z_{0}, t\right)-\left(1+\frac{z_{L}}{R_{x}}\right)\left(1+\frac{z_{L}}{R_{y}}\right) p_{z}{ }^{2} \delta w\left(x, y, z_{L}, t\right) \\
\left.+D_{z}\left(x, y, z_{0}, t\right) \delta \phi^{1}-D_{z}\left(x, y, z_{L}, t\right) \delta \phi^{n_{\phi}}\right] d A \\
-\int_{\Gamma_{L}}<\sigma_{n} \delta u_{n}+\tau_{n s} \delta u_{s}+\tau_{n z} \delta w+D_{n} \delta \phi>d s=0
\end{gathered}
$$


$\forall \delta u_{x}, \delta u_{y} \delta \mathrm{w}, \delta \phi$, where A denotes the area of the reference shell, and $\Gamma_{L}$ denotes its boundary with normal $n$ and tangent $s$.

\section{Finite element formulation}

A four-node quadrilateral element [5] based on the coupled zigzag theory is presented for the analysis of doubly curved shallow shells. As the highest derivatives of $u_{0 x}, u_{0 y}, \psi_{0 x}, \psi_{0 y}$ and $\phi_{c}^{l}$ appearing in variational Eq. (8) are of first order, the convergence criteria requires their interpolation functions to be $\mathrm{C}^{\mathbf{0}}$-continuous at the element boundaries. Accordingly, these variables are interpolated as

$$
u_{0_{x}}=N u_{0_{x}}^{e}, u_{0_{y}}=N u_{0 y}^{e}, \psi_{0_{x}}=N \psi_{0_{x}}^{e}, \psi_{0_{y}}=N \psi_{0 y}^{e}, \phi_{c}^{l}=N \phi_{c}^{l^{e}}
$$

Due to the presence of the second derivative of $w_{o}$ in Eq. (8), its interpolation function should be $\mathrm{C}^{1}$-continuous at the element boundary, which is achieved by using the improved discrete Kirchhoff (IDKQ) constraint approach, for the bending analysis of isotropic Kirchhoff plate. In this way, the rotations $\theta_{0 x}$ and $\theta_{0 y}$ are finally expressed in terms of $w_{0}^{i}, w_{0, x}^{i}\left(=\theta_{0_{x}}^{i}\right), w_{0, y}^{i}=\left(\theta_{0 y}^{i}\right)$ at the four nodes $\mathrm{i}=1,2,3,4$ as

$$
\theta_{0 x}=w_{0, x}=\mathrm{G} w_{0}^{e}, \quad \theta_{0 y}=w_{0, y}=\mathrm{H} w_{0}^{e},
$$

where,

$$
\begin{aligned}
& w_{0}^{e}=\left[\begin{array}{llllllllllll}
w_{0}^{1} & w_{0, x}^{1} & w_{0, y}^{1} & w_{0}^{2} & w_{0, x}^{2} & w_{0, y}^{2} & w_{0}^{3} & w_{0, x}^{3} & w_{0, y}^{3} & w_{0}^{4} & w_{0, x}^{4} & w_{0, y}^{4}
\end{array}\right]^{\mathrm{T}}
\end{aligned}
$$

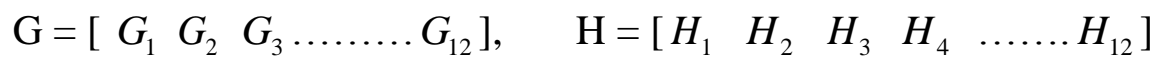

$G_{i}(\xi, \eta)$ and $H_{i}(\xi, \eta)$ are the IDKQ interpolation functions. For the purpose of computing the consistent mass matrix and the load vector, $w_{o}$ is interpolated assuming a bi-cubic function

$$
w_{0}=\left[\begin{array}{lll}
\tilde{N}_{1} & \tilde{N}_{2} & \tilde{N}_{3} \ldots \ldots \ldots . . \tilde{N}_{12}
\end{array}\right] w_{0}^{e}=\tilde{N} w_{0}^{e}
$$

where $N_{i}$ are the bi-cubic interpolation functions. The same interpolation functions were used to compute the values of $w_{0, i}$ at the element centre for imposing the constraints $\theta_{0_{i}}=w_{0, i}$ while deriving the IDKQ interpolation functions $G_{i}$ and $H_{i}$.

The generalized displacement vector $U^{e}$ of the element is defined in terms of the nodal variables $U_{i}^{e}(\mathrm{i}=1,2,3,4)$ as

$$
\begin{gathered}
U_{i}^{e}=\left[\begin{array}{cccccccc}
u_{0_{x}}^{i} & u_{0_{y}}^{i} & w_{0}^{i} & w_{0, x}^{i} & w_{0, y}^{i} & \psi_{0_{x}}^{i} & \psi_{0 y}^{i} & \phi_{c}^{l^{i}}
\end{array}\right]^{\mathrm{T}} \\
U^{e}=\left[\begin{array}{lllllll}
U_{1}^{e} & U_{2}^{e} & U_{3}^{e} & U_{4}^{e} & \phi^{j}
\end{array}\right]^{\mathrm{T}}
\end{gathered}
$$

$\bar{u}_{1}, w_{0}, \phi^{j}$ and $\bar{\varepsilon}_{i}$ are expressed as

$$
\bar{u}_{1}=N_{u} U^{e}, w_{0}=N_{w} U^{e}, \phi^{j}=N_{\phi} U^{e}, \bar{\varepsilon}_{i}=B_{i} U^{e}
$$

Substituting the generalised constitutive equations and the expressions of $\bar{\varepsilon}_{i}$, the contribution $T^{e}$ of one element can be obtained as

$$
T^{e}=\delta U^{e T}\left[\bar{M}^{e} \ddot{U}^{e}+\bar{K}^{e} U^{e}-\bar{P}^{e}\right]
$$

where $\bar{M}^{e}, \bar{K}^{e}$ and $\bar{P}^{e}$ are the element mass matrix, stiffness matrix and load vector respectively. 

motion as

Summing up the contributions of all the elements yields the system equation of

$$
\bar{M} \ddot{U}+\bar{K} U=\bar{P}
$$

where $\bar{M}, \bar{K}$ and $\bar{P}$ are assembled mass matrix, stiffness matrix and load vectors respectively. This may be partitioned as

$$
\left[\begin{array}{ccc}
M^{u u} & 0 & 0 \\
0 & 0 & 0 \\
0 & 0 & 0
\end{array}\right]\left\{\begin{array}{l}
\ddot{\bar{U}}_{\ddot{\phi}_{s}} \\
\ddot{\phi}_{a}
\end{array}\right\}+\left[\begin{array}{ccc}
K^{u u} & K^{u s} & K^{u a} \\
K^{s u} & K^{s s} & K^{s a} \\
K^{a u} & K^{a s} & K^{a a}
\end{array}\right]\left\{\begin{array}{c}
\bar{U} \\
\phi_{s} \\
\phi_{a}
\end{array}\right\}=\left\{\begin{array}{c}
\bar{P} \\
Q_{s} \\
Q_{a}
\end{array}\right\}
$$

where the vector $\bar{U}$ contains the mechanical displacement variables, $\phi_{s}$ the unknown voltages at the sensor surfaces and the intralamina potentials $\phi_{c}^{l}$ 's, and $\phi_{a}$ the actuation potentials. $M^{u u}$ and $K^{u u}$ are the electro-mechanical coupling stiffness matrices and $K^{s s}$ and $K^{a a}$ are the electric stiffness matrices. $Q_{a}$ denotes the electric load (charge) vector at actuators. $K^{\text {sa }}$ is a null matrix when the actuator and sensor layers do not share a common interface. If they have a common interface, it is grounded. In both cases, $K^{s a} \phi_{a}=0$. Using this, $\phi_{s}$ can be substituted to yield

$$
\begin{gathered}
M^{u u} \ddot{\bar{U}}+C^{u u} \dot{\bar{U}}+\bar{K}^{u u} \bar{U}=\bar{P}-K^{u a} \phi_{a} \\
\phi_{s}=-\left(K^{s s}\right)^{-1} K^{s u} \bar{U}
\end{gathered}
$$

with,

$$
\bar{K}^{u u}=K^{u u}-K^{u s}\left(K^{s s}\right)^{-1} K^{s u}
$$

For un-damped free vibration, the damping matrix $C^{u u}$ and the right-hand side vector of the above equation are set to zero. Considering that the response of the system is dominated by the first few, say m, modes, which are the most excitable ones due to lower associated energy, so that $\bar{U}$ can be approximated as

$$
\bar{U}=\sum_{i=1}^{m} \psi_{i} \hat{\eta}_{i}=\psi \eta
$$

where $\eta$ is the modal coordinate vector of first m modes and $\psi$ is the truncated modal matrix. Using this expansion, the equation of motion (Eq. (19)) can be transformed to the reduced modal space as

$$
\ddot{\eta}(t)+\Lambda \dot{\eta}(t)+\Omega \eta(t)=\tilde{P}(t)-\bar{K}^{u a} \phi_{a}
$$

where $\Lambda=\psi^{T} C^{u u} \psi, \quad \Omega=\psi^{T} \bar{K}^{u a} \psi, \tilde{P}=\psi^{T} \bar{P}$ and $\bar{K}^{u a}=\psi^{T} K^{u a}$. The system of second order ordinary differential equations given by Eq. (21) is expressed in the state space form involving first order ordinary differential equations as

$$
\begin{gathered}
\dot{X}(t)=A X(t)+B_{u} u_{u}(t)+B_{\phi} u_{\phi}(t) \\
Y(t)=C X(t)
\end{gathered}
$$

where $X(t)=\operatorname{col}(\eta, \dot{\eta})$ and $Y(t)=\operatorname{col}\left(\phi_{m}, \delta_{\mathrm{c}} \dot{\phi}_{m}\right)$ are the state vector and the output vector respectively. A is the system state matrix, and $B_{u}$ and $B_{\phi}$ are are the mechanical and electrical input matrices, $\mathrm{C}$ is the output matrix, and $\mathrm{u}_{u}(\mathrm{t})$ and $\mathrm{u}_{\phi}(\mathrm{t})$ are the mechanical excitations and electrical control input vectors respectively. 


\section{Feedback control laws}

In the constant gain velocity feedback (CGVF) control, the control input voltage is taken as

$$
\mathrm{u}_{\phi}(t)=-G Y(t)=-G C X(t)
$$

with $\mathrm{G}=\left[\begin{array}{ll}0 & G_{v}\end{array}\right]$, where $G_{v}$ is the velocity gain matrix. Substitution of Eq. (23) into Eq. (22) essentially leads to modification of the state matrix through an additional active damping matrix $\Lambda_{a}=\psi^{\mathrm{T}} \mathrm{K}^{u a} \quad G_{v} C_{m}\left[\mathrm{~K}^{s s}\right]^{-1} \mathrm{~K}^{s u} \psi$. The asymptotic stability of the response depends on the positive definiteness of $\Lambda_{a}$. For truly collocated actuator-sensor pairs, $\Lambda_{a}$ will be symmetric positive definite, ensuring asymptotic stability of the response.

In optimal control, the feedback control gains are determined by minimizing the performance index or cost function, defined as a quadratic function of the control input and the measured output as follows

$$
J=\int_{o}^{\infty}\left[Y^{T}(t) Q_{Y} Y(t)+\mathrm{u}_{\phi}^{T}(t) R \mathrm{u}_{\phi}(t)\right] d t
$$

where $Q_{Y}$ and $R$ are the output and control input weighing matrices, which should be real symmetric positive definite. For a steady state LQR controller, the control input is given by optimal state feedback matrix $G\left[\mathrm{u}_{\phi}(t)=-G X(t)\right]$, which is obtained as

$$
G=[R]^{-1} \mathrm{~B}_{\phi}{ }^{T} P
$$

where $\mathrm{P}$ is the solution of the following steady state Ricatti equation

$$
A^{T} P+P A-P \mathrm{~B}_{\phi} R^{-1} \mathrm{~B}_{\phi}{ }^{T} P+C^{T} Q_{Y} C=0
$$

\section{Integrated approach}

An integrated approach is used for recording the uncontrolled and controlled vibration response under pressure impulse load. The FE models of smart composite shallow shells with prescribed loading and boundary conditions are developed in ABAQUS which are than utilised to generate the global mass, stiffness and load matrices of the system. The global matrices are partitioned to create $M^{u u}, K^{u u}, K^{u a}, K^{a a}, K^{a u}$ matrices and the load vector. The system matrices are arranged in state-space format. These inputs from FE formulation in ABAQUS are used in conjunction with the developed MATLAB codes to obtain the free vibration responses. Active control is achieved using Constant gain velocity feedback (CGVF) and Linear Quadratic Regulator (LQR) as the control strategies.

For generating the finite element mesh in ABAQUS, an 8-noded linear piezoelectric brick element (C3D8E) is used for piezo layers and an 8-noded quadrilateral in-plane general-purpose continuum shell element (SC8R) is used for the elastic layers of hybrid shells. The geometric order is considered linear for both type of elements. Continuum shell elements have only displacement degrees of freedom. From a modelling point of view continuum shell elements look like three-dimensional continuum solids, but their kinematic and constitutive behaviour is similar to conventional shell elements. Mechanical nodes, corresponding to elastic layers, have 3 degrees of freedom (i.e. translations along $\mathrm{x}, \mathrm{y}, \mathrm{z}$ directions) and piezo nodes, corresponding to piezoelectric layers, have 4 degrees of freedom (i.e. 3 mechanical and 1 electrical). 


\section{RESULTS AND DISCUSSION}

A hybrid composite shallow spherical shell $\left(R_{x} / a=2, R_{y} / b=2\right)$ of square planform and a cylindrical shell $\left(R_{x} / a=2, R_{y} / b=\infty\right)$ of rectangular planform for different span to thickness ratios, $S$, are analysed here. Figure 1 shows composite lamina configuration for hybrid composite shells. The elastic substrate is a graphite-epoxy (Gr/Ep-1) composite laminate with 3 layers of equal thicknesses $0.3 h$ with lay-up $\left(0^{\circ} / 90^{\circ} / 0^{\circ}\right)$. Both the shells have a PZT-5A layer of thickness $0.1 \mathrm{~h}$ bonded to the top of the elastic substrate. The PZT-5A layer have polling in $+z$ direction. The interfaces of the PZT layers with the elastic substrate are grounded. The top surface of PZT-5A layer is subjected to open circuit condition.

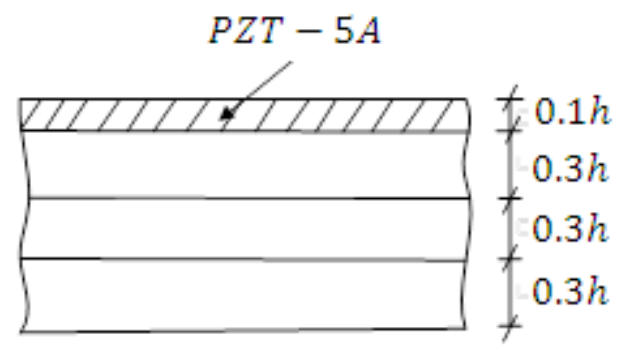

Figure 1. Composite lamina configuration for hybrid composite shells.

The properties of materials used are given by:

$\left[Y_{1}, Y_{2}, Y_{3}, G_{12}, G_{23}, G_{31},{ }_{12}, v_{13}, v_{23, \rho}\right]$

Gr/Ep-1: $\left[(172.5,6.9,6.9,3.45,1.38,3.45) \mathrm{GPa}, 0.25,0.25,0.25,(1600) \mathrm{kg} / \mathrm{m}^{3}\right]$

PZT-5A: $\left[(61.0,53.2,61.0,21.1,21.1,22.6) \mathrm{GPa}, 0.38,0.38,0.35,(7600) \mathrm{kg} / \mathrm{m}^{3}\right]$

$\left[\left(d_{15}, d_{31}, d_{33}, d_{32}, d_{24}\right),\left(D_{11}, D_{22}, D_{33},\right)\right.$

$=\left[(584,-171,374,-171,584) \times 10^{-12} \mathrm{~m} / \mathrm{V},(1.53,1.53,1.53) \times 10^{-8} \mathrm{~F} / \mathrm{m}\right]$.

\section{Free Vibration Response}

The natural frequencies of hybrid composite cylindrical and spherical shells for free vibration response are obtained using integrated approach and compared with corresponding FE results obtained through efficient zigzag theory (Table 1 and 2).

Table 1. Non-dimensional natural frequencies of hybrid composite spherical shell.

\begin{tabular}{cccccc}
\hline \multicolumn{2}{c}{ Natural frequency, $\bar{\omega}_{n}$} \\
\hline \multicolumn{5}{c}{ Cantilever } & \multicolumn{2}{c}{ Clamped-Clamped } \\
\hline \multirow{2}{*}{ S } & $\begin{array}{c}\text { Mode } \\
\text { No. }\end{array}$ & $\begin{array}{c}\text { Integrated } \\
\text { Approach }\end{array}$ & FE- Zigzag & $\begin{array}{c}\text { Integrated } \\
\text { Approach }\end{array}$ & FE- Zigzag \\
\hline \multirow{4}{*}{10} & 1 & 3.036 & 3.035 & 8.079 & 8.075 \\
& 2 & 4.327 & 4.324 & 14.331 & 14.324 \\
& 3 & 7.580 & 7.576 & 15.764 & 15.756 \\
& 4 & 14.339 & 14.332 & 17.929 & 17.920 \\
& 5 & 14.831 & 14.824 & 18.443 & 18.433 \\
\hline \multirow{4}{*}{20} & 1 & 5.365 & 5.363 & 20.329 & 20.319 \\
& 2 & 6.553 & 6.550 & 29.555 & 29.539 \\
& 3 & 17.785 & 17.776 & 31.073 & 31.056 \\
& 4 & 22.618 & 22.607 & 31.634 & 31.617 \\
\hline \hline & 5 & 28.415 & 28.401 & 36.301 & 36.283 \\
\hline
\end{tabular}


The frequencies have been non-dimensionalized as follows:

$$
\bar{\omega}_{n}=\omega_{n} * a * \mathrm{~S} * \sqrt{\frac{\rho}{E_{2}}}
$$

The natural frequencies have been presented for cantilever and clamped-clamped boundary conditions for different span to thickness ratios, $S$. As observed from the tables, the results have been found in good agreement. A general increasing trend with increasing mode number has been observed. As expected, the natural frequencies of a shell of given geometry, configuration and boundary conditions increases with span to thickness ratio.

Table 2. Non-dimensional natural frequencies of hybrid composite cylindrical shell.

\begin{tabular}{cccccc}
\hline \multicolumn{2}{c}{ Natural frequency, $\bar{\omega}_{n}$} \\
\hline \multicolumn{5}{c}{ Cantilever } & \multicolumn{2}{c}{ Clamped-Clamped } \\
\hline \multirow{2}{*}{$\mathrm{S}$} & \multirow{2}{*}{ Mode } & $\begin{array}{c}\text { Integrated } \\
\text { Approach }\end{array}$ & FE- Zigzag & $\begin{array}{c}\text { Integrated } \\
\text { Approach }\end{array}$ & FE- Zigzag \\
\hline \multirow{2}{*}{10} & 1 & 0.733 & 0.733 & 3.543 & 3.539 \\
& 2 & 1.172 & 1.169 & 3.747 & 3.739 \\
& 3 & 1.355 & 1.352 & 6.103 & 6.083 \\
& 4 & 3.749 & 3.746 & 8.861 & 8.836 \\
& 5 & 3.916 & 3.912 & 10.515 & 10.490 \\
\hline 20 & 1 & 1.357 & 1.356 & 6.041 & 6.038 \\
& 2 & 1.772 & 1.771 & 6.122 & 6.119 \\
& 3 & 2.541 & 2.539 & 11.168 & 11.162 \\
& 4 & 6.206 & 6.203 & 15.729 & 15.720 \\
& 5 & 6.231 & 6.228 & 20.236 & 20.225 \\
\hline \hline
\end{tabular}

\section{Active Vibration Control}

The active vibration control results using integrated approach are obtained for hybrid composite cylindrical and spherical shells for cantilever and clamped-clamped boundary conditions. The results are presented for two values of span to thickness ratios $(S)$ viz. 10 and 20, for constant gain velocity feedback (CGVF) control and Linear Quadratic Regulator (LQR) control. The shells are excited by a $1 \mathrm{kN}$ pressure impulse load. The uncontrolled and controlled responses obtained by the integrated approach are shown in Figure 2 to Figure 9. The effect of geometry of shells (cylindrical or spherical), boundary conditions and span to thickness ratio $(S=a / h)$ on the vibration control characteristics can be observed from these figures.

As observed for cantilever composite cylindrical shell (Figure 2) for span to thickness $\operatorname{ratio}(S)=10$, the vibration control time using CGVF control is approximately 5 seconds and the maximum amplitude of control voltage is $10 \mathrm{~V}$ and a peak is observed at frequency $2.5 \mathrm{~Hz}$ (Figure 8). The vibration control time increases to about 12 seconds and maximum amplitude of control voltage to around 40 volts as the shell becomes 
thinner $(S=20)$. Further, the gain value $(G)$ required for vibration control for thick shells is more.

For the same controller type and span to thickness ratio $(S)$, as the boundary condition is changed to clamped-clamped, the maximum amplitude of control voltage increases and the control time decreases (Figure 3). In comparison to CGVF controller (Figure 3), a better control in lesser time is achieved with LQR controller for the same shell with similar boundary conditions (Figure 4). Similar observations are obtained by comparing Figure 2 and Figure 7.
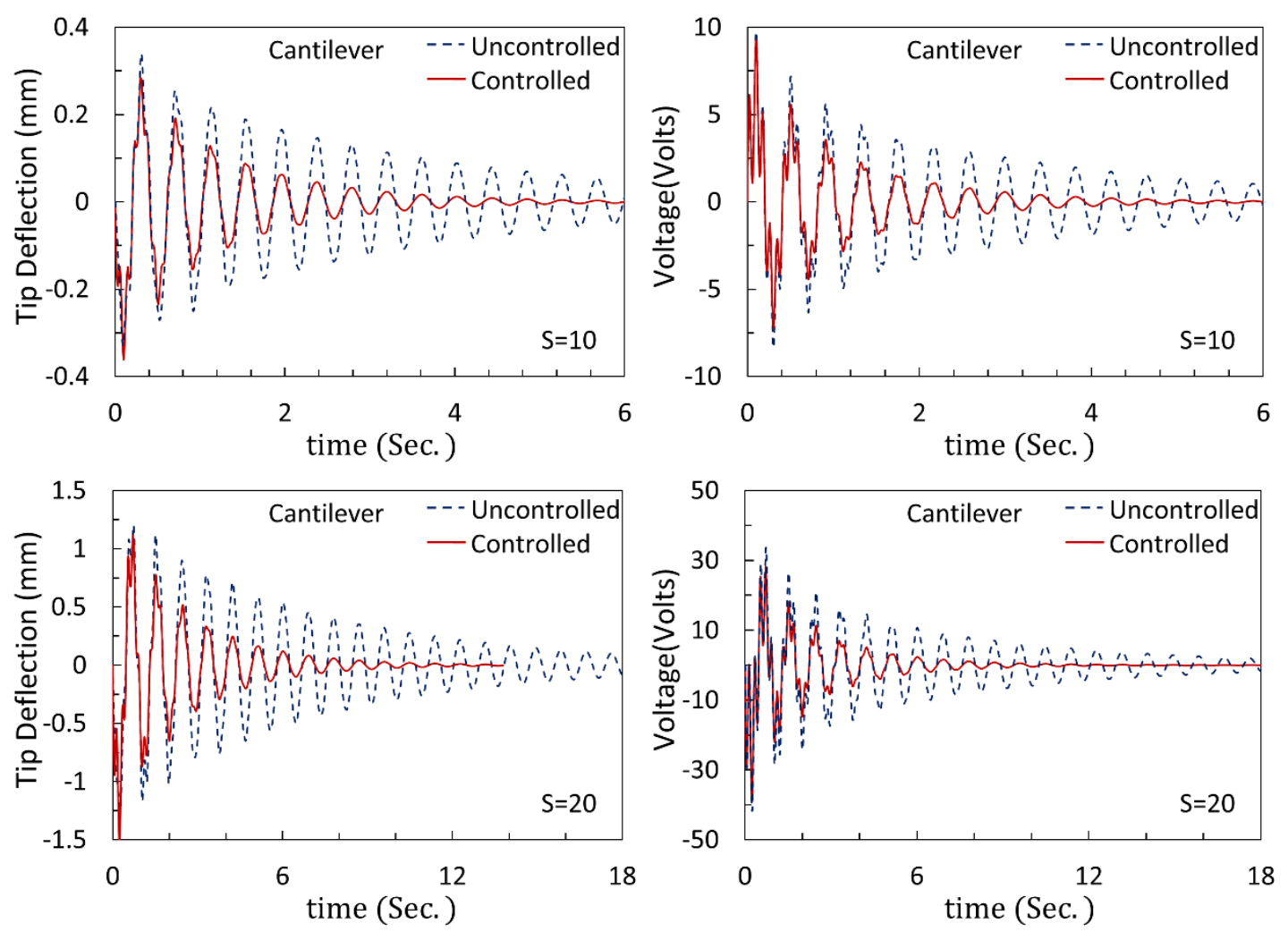

Figure 2. Tip deflection and corresponding voltage for hybrid composite cylindrical shell subjected to pressure impulse load under CGVF control.
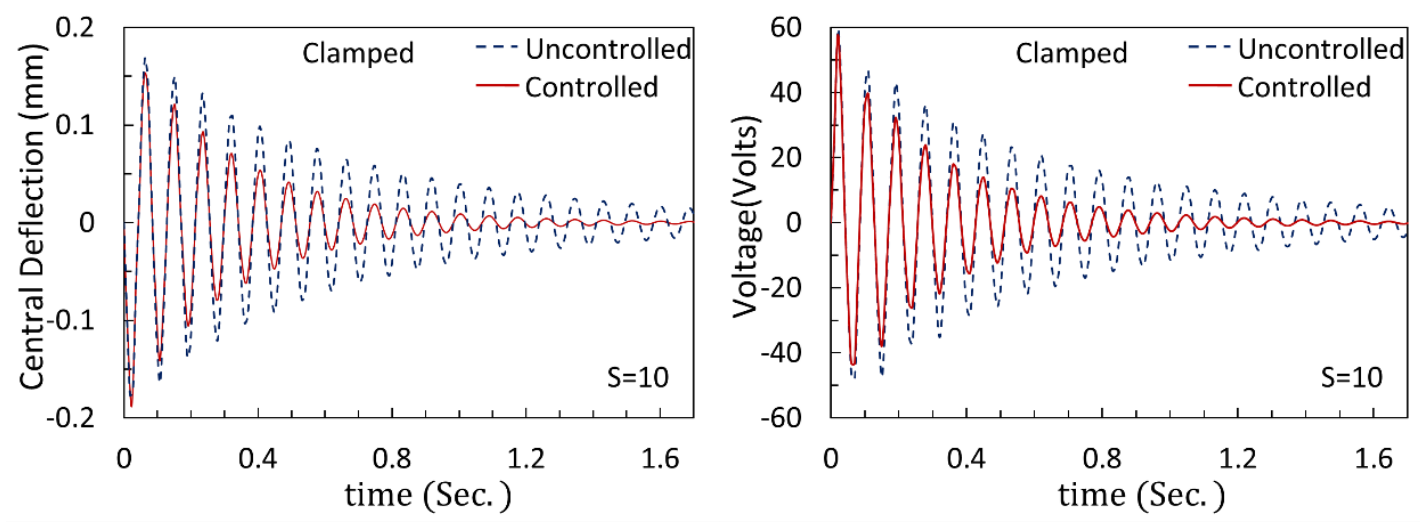

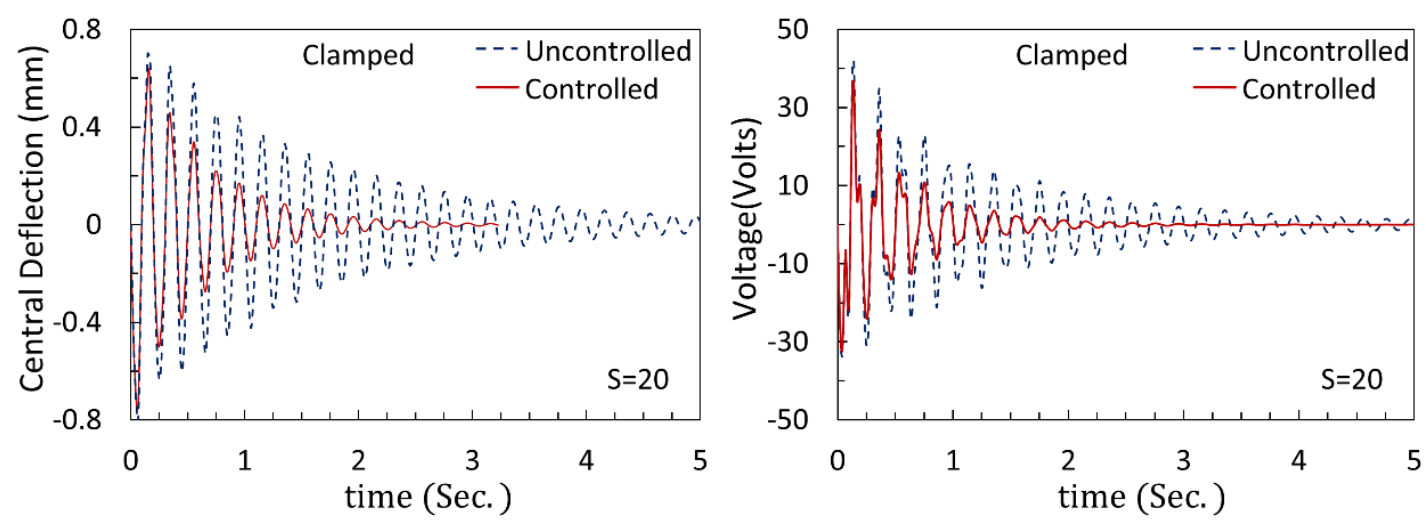

Figure 3. Centre deflection and corresponding voltage for hybrid composite cylindrical shell subjected to pressure impulse load under CGVF control.
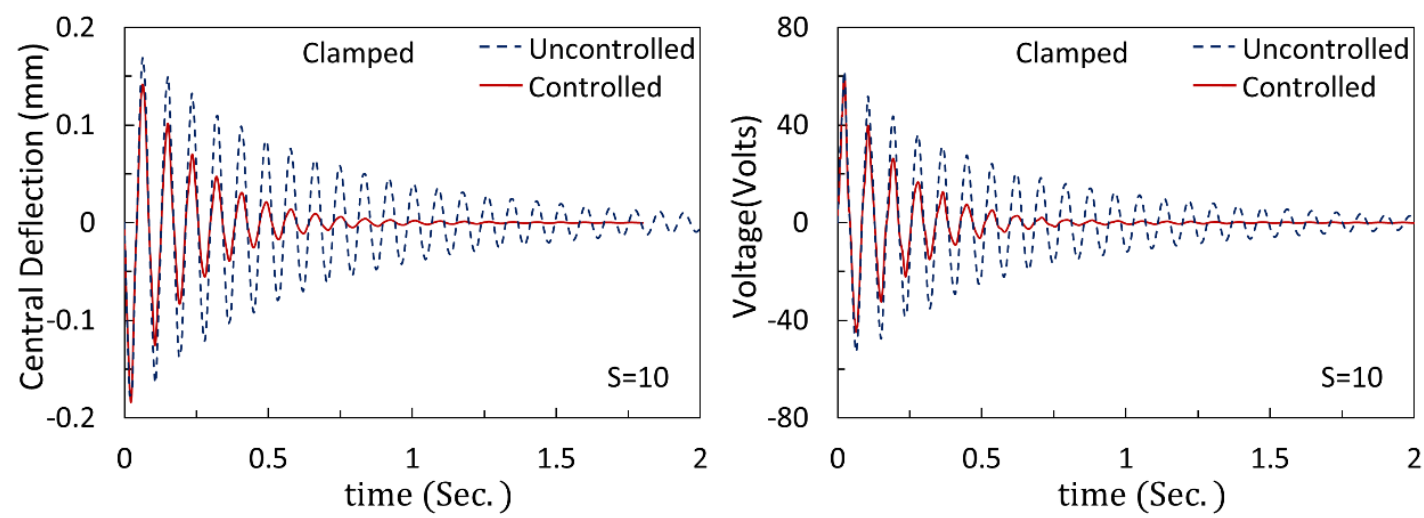

Figure 4. Centre deflection and corresponding voltage for hybrid composite cylindrical shell subjected to pressure impulse load under LQR control.

Figure 5 shows tip deflection and corresponding voltage for cantilever hybrid composite spherical shell subjected to pressure impulse load with CGVF control. The vibration control time, for span to thickness $\operatorname{ratio}(S)=10$, is approximately 1.5 seconds and the maximum amplitude of control voltage is $6 \mathrm{~V}$. These parameters are respectively 2 seconds and $90 \mathrm{~V}$ for $S=20$. The frequency response plots (Figure 6) show the comparison for these two values of span to thickness ratios. 

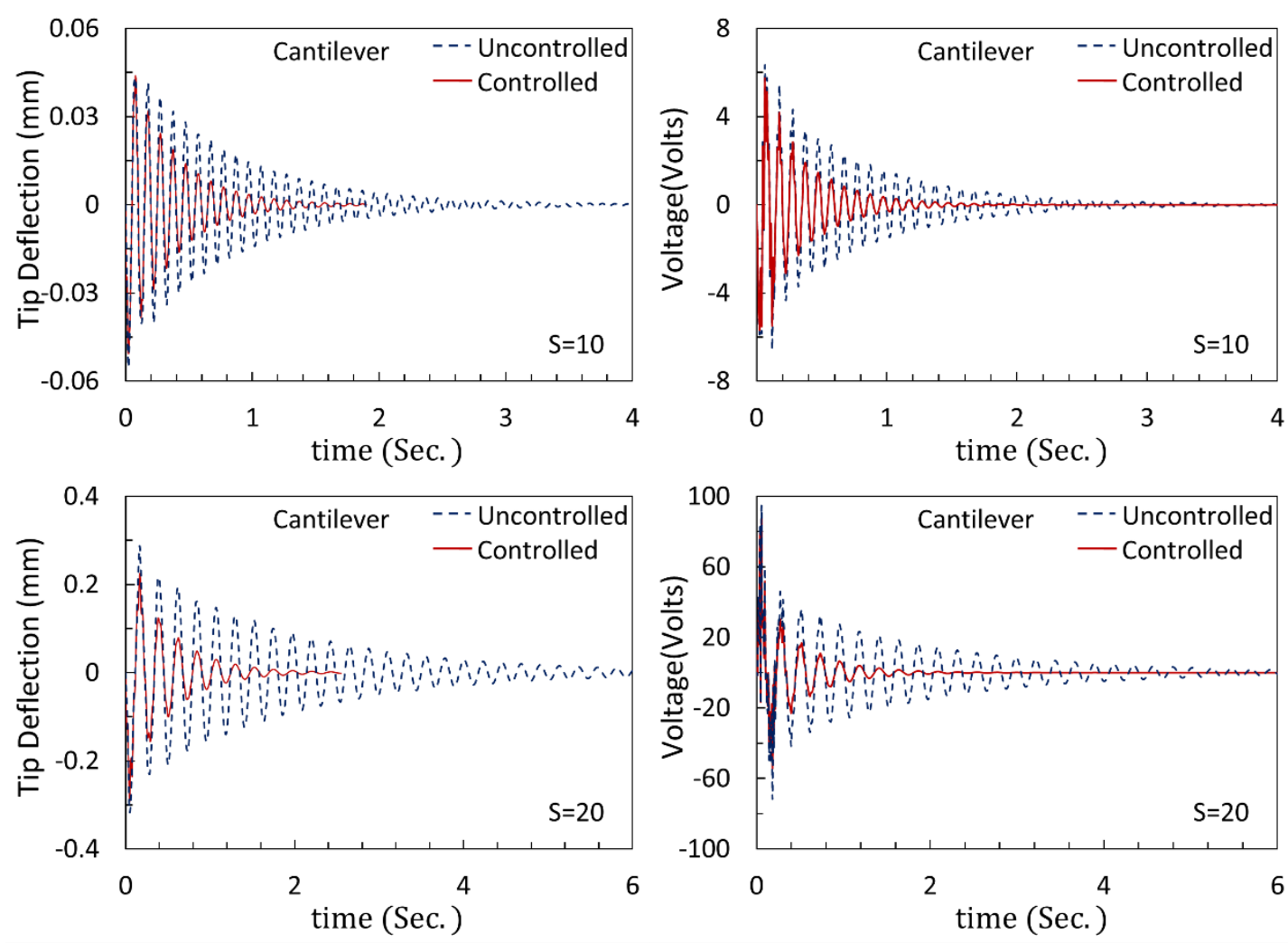

Figure 5. Tip deflection and corresponding voltage for hybrid composite spherical shell subjected to pressure impulse load under CGVF control.
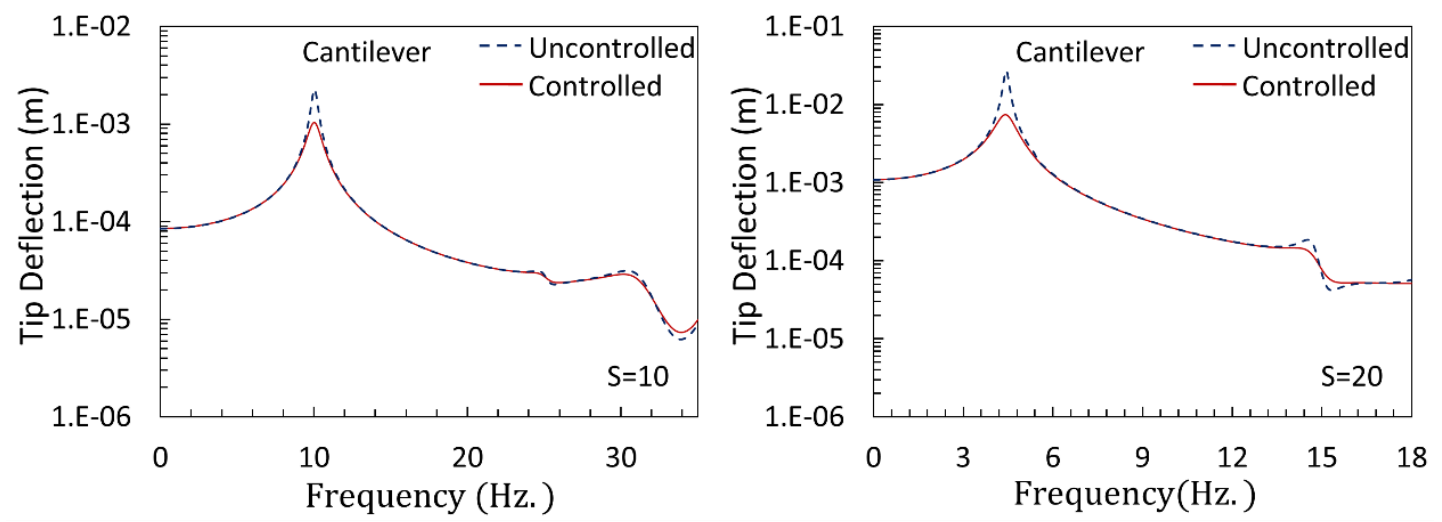

Figure 6. Frequency response for tip deflection of hybrid composite spherical shell subjected to pressure impulse load under CGVF control. 

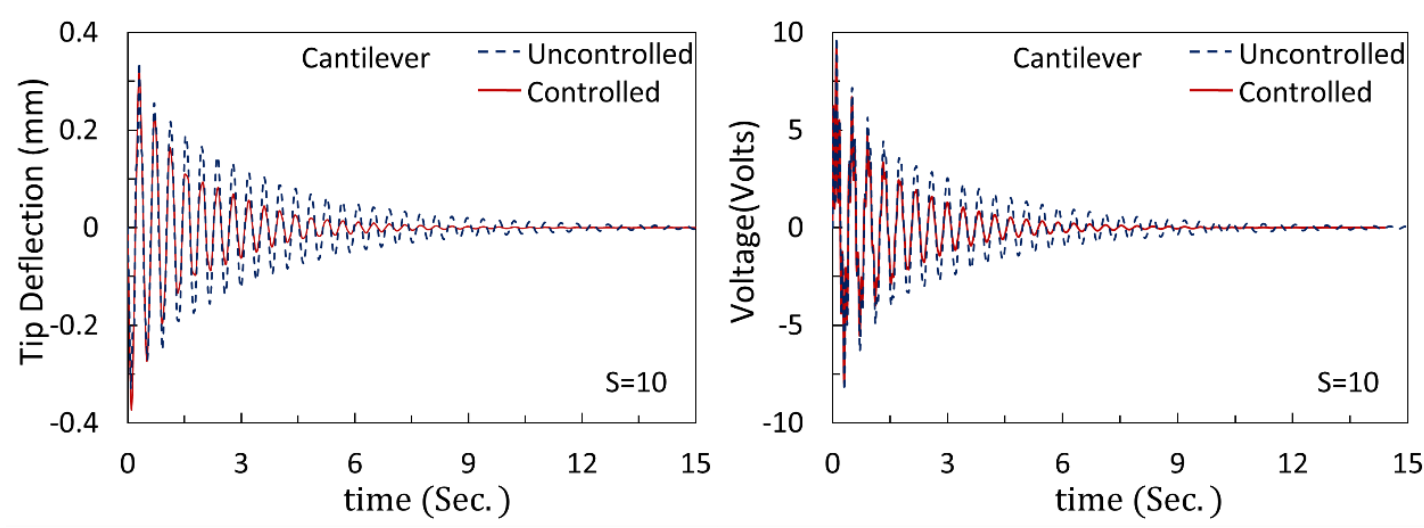

Figure 7. Tip deflection and corresponding voltage for hybrid composite cylindrical shell subjected to pressure impulse load under LQR control.
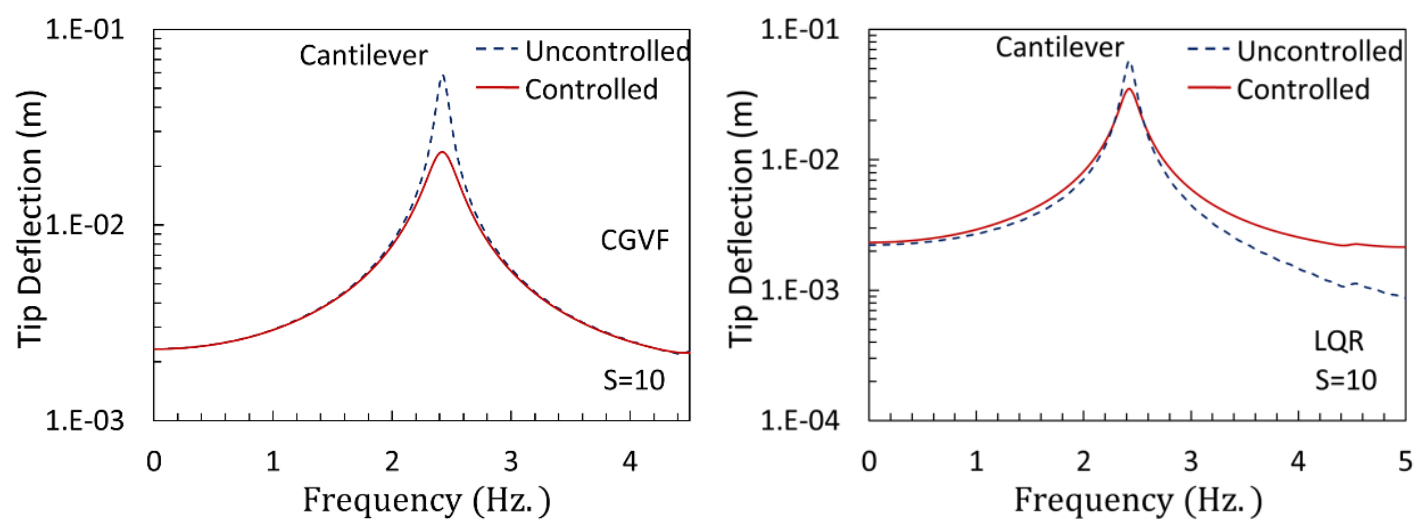

Figure 8 . Frequency response for tip deflection of hybrid composite cylindrical shell subjected to pressure impulse load.

As the span to thickness ratio and boundary conditions remain same (Figure 8), the peak is obtained at about $2.5 \mathrm{~Hz}$ in the frequency response plot of hybrid composite cylindrical shell for both CGVF and LQR controllers.

The integrated approach is simpler to use as it saves lots of computational efforts. This methodology eliminates the theoretical geometric modelling without compromising accuracy. It is simple to model complex structures in commercial FE package ABAQUS. The inputs from this FE model can be efficiently used to generate control algorithms in MATLAB environment. The results presented reveal that early attainment of vibration suppression is achieved using optimal LQR controller. Since spherical shells are doubly curved, the given excitation produces smaller amplitude and hence lesser settling time is required for vibration suppression. As expected, for the thin shells control time is more because the amplitude of vibration is higher as compare to thick shells. As a result the gain value $(G)$ required for vibration control for thick shells is more. Boundary conditions also have significant effect on vibration amplitude, control voltage and settling time. 

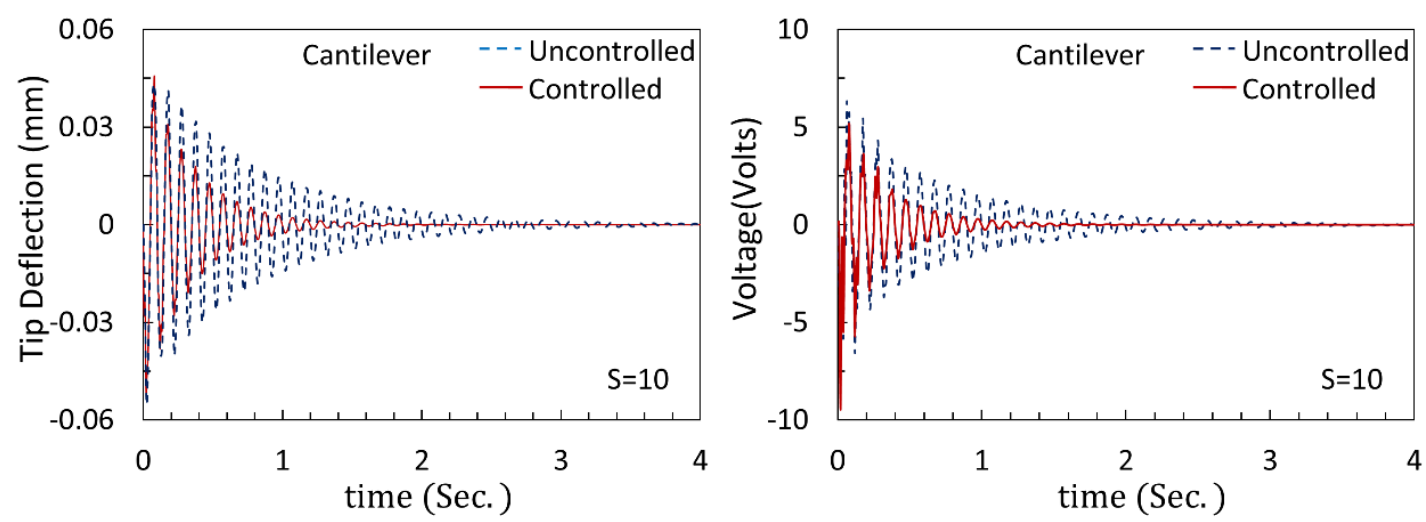

Figure 9. Tip deflection and corresponding voltage for hybrid composite spherical shell subjected to pressure impulse load under LQR control.

\section{CONCLUSIONS}

The active vibration control response of hybrid composite shallow shells is studied using an integrated approach. The model is able to analyse the hybrid shell with good computational efficiency. The controlled responses are obtained using constant gain velocity feedback (CGVF) and Linear Quadratic Regulator (LQR) control strategies. The problem considered reveals the following important findings.

The optimal LQR controller is better than the conventional CGVF controller as it give early attainment of vibration suppression. The spherical shell has smaller amplitude and require lesser time to control the vibrations in comparison to the cylindrical shell for the same excitation. This is because the spherical shell is doubly curved. The vibration control time increases as the shell becomes thinner. Further, the gain value $(G)$ required for vibration control for thick shells is more. The boundary conditions have significant effect on vibration amplitude, control voltage and settling time. For similar situations, as the boundary condition is changed from cantilever to clamped-clamped, the maximum amplitude of control voltage increases and the control time decreases. For the case of free vibrations under either end conditions, the natural frequencies are higher for higher modes of vibration. As the shell is made thinner for a given shell configuration and boundary conditions, the natural frequencies tend to increase.

The methodology presented is simple and can be implemented with various active vibration control strategies for complex structures.

\section{REFERENCES}

[1] Qian W, Liu GR, Chun L, \& Lam KY. Active vibration control of composite laminated cylindrical shells via surface-bonded magnetostrictive layers. Smart Materials \& Structures. 2003;12(6):889-897

[2] Bhattacharya P, Suhail H \& Sinha PK. Finite element analysis and distributed control of laminated composite shells using LQR/IMSC approach. Aerospace Science and Technology. 2002; 6:273-281.

[3] Balamurugan V \& Narayanan S. A piezolaminated composite degenerated shell finite element for active control of structures with distributed piezosensors and actuators. Smart Materials \& Structures.2008; 17(3):1-18.

[4] Ray M. C. Optimal control of laminated shells using piezoelectric sensor and actuator layers. AIAA Journal. 2003; 41(6):1151-1157. 
[5] Kapuria S \& Yasin MY. Coupled efficient layerwise finite element modeling for active vibration control of smart composite and sandwich shallow shells. Journal of Intelligent Material Systems and Structures. 2014; 25(16):2013-2036.

[6] Ray MC \& Reddy JN. Optimal control of thin circular cylindrical laminated composite shells using active constrained layer damping treatment. Smart Materials \& Structures. 2004; 13(1): 64-72.

[7] Kumar DN, Raja S \& Ikeda T. Active vibration control of smart plates with partially debonded multi-layered PZT actuators. Smart Mater Struct. 2007; 16: 1584-1594.

[8] Zhang YH, Xie SL \& Zhang XN. Vibration control of a simply supported cylindrical shell using a laminated piezoelectric actuator. Acta Mechanica. 2008; 196(1-2): 87-101.

[9] Rahman N \& Alam MN. Active vibration control of a piezoelectric beam using PID controller: Experimental study. Latin American Journal of Solids and Structures.2012; 9(6):657-673.

[10] Li HY, Lin QY, Liu ZX \& Wang C. Active control of the piezoelastic laminated cylindrical shell's vibration under hydrostatic pressure. Applied Mathematics and Mechanics-English Edition. 2003; 24(2):182-195.

[11] Kapuria S \& Yasin MY. Active vibration control of piezoelectric laminated beams with electroded actuators and sensors using an efficient finite element involving an electric node. Smart Materials and Structures. 2010; 19(4). doi: Artn 045019.

[12] Lee SJ and Reddy JN Vibration suppression of laminated shell structures investigated using higher order shear deformation theory. Smart Materials \& Structures. 2004; 13(5):1176-1194.

[13] Rahman N and Alam MN. Active Vibration Suppression of Piezoelectric Laminated Beam based on Efficient Finite Element Model using LQG Controller. International Journal of Theoretical and Applied Mechanics. 2012; 7(1): 9-24.

[14] Rahman N and Alam MN. Structural Control of Piezoelectric Laminated Beams under Thermal Load. Journal of Thermal Stresse.2015; 38: 69-95.

[15] Moita JMS, Correia IFP, Soares CMM \& Soares CAM. Active control of adaptive laminated structures with bonded piezoelectric sensors and actuators. Computers \& Structures. 2004; 82(17-19): 1349-1358.

[16] Ray MC \& Mallik N. Active control of laminated composite beams using a piezoelectric fibre reinforced composite layer. Smart Mater Struct. 2004; 13: 146-152.

[17] Pradhan SC \& Reddy JN. Vibration control of composite shells using embedded actuating layers. Smart Materials \& Structures.2004; 13(5): 1245-1257.

[18] Ray MC \& Batra RC. Smart constrained layer damping of functionally graded shells using vertically/obliquely reinforced 1-3 piezocomposite under a thermal environment. Smart Materials Structures. 2008; 17(5). doi: Artn 055007 10.1088/0964-1726/17/5/055007

[19] Zhang YH, Zhang XN \& Xie SL. Adaptive vibration control of a cylindrical shell with laminated PVDF actuator. Acta Mechanica. 2010; 210(1-2): 85-98.

[20] Sarangi SK \& Ray MC. Smart control of nonlinear vibrations of doubly curved functionally graded laminated composite shells under a thermal environment using 1-3 piezoelectric composites. International Journal of Mechanics and Materials in Design. 2013; 9(3): 253-280. 
[21] Neto MA, Ambrosio JAC, Roseiro LM, Amaro A \& Vasques CMA. Active vibration control of spatial flexible mutibody systems. Multibody Syst Dyn.2013; 30(1):13-35.

[22] Hua-ping T, Yun-jun T \& Gong-an T. Active vibration control of mutibody system with quick startup and brake based on active damping. J Cent South Univ T. 2006; 13(4): 417- 421.

[23] Song ZG \& Li FM. Active aeroelastic flutter analysis and vibration control of supersonic composite laminated plate. Composite Structures. 2012; 94(2): 702713.

[24] Shah PH \& Ray MC. Active control of laminated composite truncated conical shells using vertically and obliquely reinforced 1-3 piezoelectric composites. European Journal of Mechanics a-Solids. 2012; 32: 1-12.

[25] Sarangi SK \& Ray MC. Active damping of geometrically nonlinear vibrations of doubly curved laminated composite shells. Composite Structures. 2011; 93(12):3216-3228.

[26] Sun BH \& Huang D. Vibration suppression of laminated composite beams with a piezo-electric damping layer. Composite Structures. 2001; 53(4): 437-447.

[27] Zhang HY \& Shen YP. Vibration suppression of laminated plates with 1-3 piezoelectric fiber-reinforced composite layers equipped with interdigitated electrodes. Composite Structures. 2007; 79(2): 220-228.

[28] Baillargeon BP, Vel SS \& Koplik JS. Utilizing ABAQUS to Analyze the Active Vibration Suppression of Structural Systems. Paper presented at the ABAQUS Users'Conference, Boston, MA USA. 2004.

[29] Vasques CMA \& Rodrigues JD. Active vibration control of smart piezoelectric beams: Comparison of classical and optimal feedback control strategies. Computers \& Structures. 2006; 84(22-23): 1402-1414.

[30] Balamurugan V \& Narayanan S. Shell finite element for smart piezoelectric composite plate/shell structures and its application to the study of active vibration control. Finite Elem Anal Des. 2001; 37(9): 713-738.

[31] Roy T \& Chakraborty D. (2009). Optimal vibration control of smart fiber reinforced composite shell structures using improved genetic algorithm. Journal of Sound and Vibration. 2009; 319(1-2): 15-40. doi: 10.1016/j.jsv.2008.05.037

[32] Bailey T \& Hubbard JE (1985). Distributed Piezoelectric Polymer Active Vibration Control of a Cantilever Beam. Journal of Guidance Control and Dynamics. 1985; 8(5): 605-611. doi: Doi 10.2514/3.20029

[33] Kim SJ, Hwang JS \& Mok J. Active vibration control of composite shell structure using modal Sensor/Actuator system. KSAS International Journal. 2006; 7(1): 106-117.

[34] Sun L, Li W, Wu Y and Lan Q. Active vibration control of a conical shell using piezoelectric ceramics. Journal of Low Frequency Noise,Vibration and Active Control. 2017; 36(4): 366-375

[35] Loghmani A, Danesh M, Keshmiri $M$ and Savadi MM. Theoretical and experimental study of active vibration control of a cylindrical shell using piezoelectric disks. Journal of Low Frequency Noise,Vibration and Active Control. 2015; 34(3): $269-288$

[36] He J and Chen X. Integrated topology optimization of structure/vibration control for piezoelectric cylindrical shell based on the genetic algorithm. Shock and Vibration. 2015; 2015: 10. doi: 10.1155/2015/456147. 